\title{
On the Application of Background Music in University Library
}

\author{
Hui Yang, Kongguo Zhu*, Shulong Li
}

Library, Shandong Youth University of Political Science, Ji'nan 250103, China

${ }^{*}$ Corresponding author. Email: zhukongguo@126.com

\begin{abstract}
University library is one of the main places for college students to study. The integration of background music and library services can achieve unexpected results. The correct embedding of music elements can reduce the fatigue of teachers and students in their work and study, relieve the bad emotions caused by the pressure of life, improve the efficiency of study and work, and improve their musical quality and artistic attainments.
\end{abstract}

Keywords: University Library, Background Music, Music Styles, Service Innovation.

\section{背景音乐在高校图书馆的运用探析}

\author{
杨慧, 朱孔国*, 李淑龙
}

山东青年政治学院图书馆, 济南 250103 中国

*通讯作者. 邮箱: zhukongguo@126.com

\section{中文摘要}

高校图书馆是大学生学习的主要场所之一，将背景音乐与图书馆的各项服务相融合能收到意想不到的效果。正 确地嵌入音乐元素能够减轻师生工作及学习产生的疲劳感, 缓解由生活压力等产生的不良情绪, 提升学习及工 作效率，提高音乐素养和艺术造诣。

关键词：高校图书馆，背景音乐，音乐类型，服务创新

\section{1. 简介}

音乐是人类文化传承的重要载体，其独有的艺术 魅力不仅能培养人的审美气质, 亦能使人们的心绪在 音乐中得以释放, 是人类不可或缺的精神食粮。众所 周知, 背景音乐已广泛应用于商场、餐厅、超市、书 城等场所, 在很多文化场所, 如美术馆、博物馆、展 览馆等也常播放舒缓优雅的背景音乐, 很多高校图书 馆也有应用, 只是还远未达到普及的程度。

高校图书馆是读者集中学习与阅览的活动场所, 适当的背景音乐能消除一定的心理疲劳、缓解心理压 力、稳定心绪。舒缓轻柔的背景音乐完全不同于噪音,
恰当的背景音乐反能一定程度上覆盖噪音, 增强图书 馆的亲和力, 营造良好的学习氛围。西密歇根大学图 书馆员的定性和定量研究表明, 背景音乐与学生的舒 适感、自信心和记忆力呈正相关【1】。针对这个问 题, 对在校读者做了 149 份有关高校图书馆背景音乐 的调查问卷, 结果显示有 $75.17 \%$ 的读者支持在开馆、 闭馆和午休时间短时间地播放背景音乐，有 $50.34 \%$ 读者经常边听音乐边看书, 这足以证明了读者对背景 音乐的需求。 


\section{2. 背景音乐的作用和功能}

\section{1. 缓解读者大脑疲劳、提高学习效率}

据研究，音乐能一定程度刺激脑神经、调节脑波、 缓解大脑疲劳; 亦能开发智能, 激发灵感, 活跃思维, 提高学习效率。和谐唯美的音乐可刺激右半脑, 使大 脑放松, 同时又能促进左脑思维能力的提高, 使思维 更加敏捷, 可促进体内蛋白质合成以及其它大脑化学 变化, 加速学习和记忆进程。

世界著名心理学专家保加利亚 lozanov 博士曾对 背景音乐对学习影响方面进行了长期而颇有成效的 研究, 他认为, 音乐能使人注意力更加集中, 消除烦 躁, 有利于心绪稳定。背景音乐同时也能一定程度上 消除单一思维的长期状态而产生的疲劳, 可给予读者 变化的刺激，提高学习效率【2】。

\section{2. 缓解大学生日益突出的心理问题}

当今时代, 大学生学习就业压力很大, 苦闷、焦 虑、抑郁、孤独等心理问题凸显, 心理问题严重者甚 至会导致恶劣后果。近年来, 高校大学生、博士生跳 楼自杀案例时有发生, 南京大学在读女博士 2020 年 9 月份宿舍跳楼自杀; 10 月份, 大连理工研究生 6 楼卫 生间坠楼身亡, 江苏大学湖北籍大学生跳楼身亡”, 南京审计大学跳楼身亡.......... 这一个个鲜活生命的坠 亡使人悲痛, 学会适度释放情绪与压力, 提升“抗压 能力”颇为重要。图书馆是高校德育教育的重要场所, 舒缓优美背景音乐可使读者在紧张的压力下适度缓 解紧张焦虑情绪, 提升自我行为掌控能力, 不同的音 乐可以使读者产生不同的生理反应和心理情趣, 但都 可通过优美的音乐使人产生积极乐观的情绪和想象。

\section{3. 增强图书馆亲和力，营造良好学习阅览氛 围}

高校图书馆作为高校三大支柱之一，作为文化资 源聚集地, 素来以“静”字要求读者, 很多高校图书馆 张贴“请勿朗读、严禁喧哗”的警示牌。但背景音乐全 然不是噪音, 相反, 轻柔舒缓的音乐能一定程度上掩 盖低语声、脚步声、拖动桌椅声等无可避免的噪音, 且能给读者带来享受音乐的快感, 增强图书馆亲和力。 调查中 $64.43 \%$ 的读者认为在图书馆说话声最容易分 散注意力。2 $7.52 \%$ 读者认为其他噪音最容易分散注意 力, $8.05 \%$ 的读者认为背景音乐容易分散注意力。适 当的背景音乐不仅能给单调的学习和阅览增添了优 雅的格调, 提高学习效率, 亦无形中优化了学习阅览 环境, 给读者一种美的感受, 提升图书馆的服务形象。

\section{3. 背景音乐的使用和选择}

\section{1. 背景音乐的乐曲选择}

高校图书馆背景音乐需融入整个环境氛围中, 结 合不同时间段、节日、季节等时间因素和乐曲的音乐 特点来选择背景音乐。一般适宜选择一些舒缓的轻音 乐, 因为舒缓的轻音乐节奏与人的脉动的节律比较吻 合, 舒缓音乐会让读者感到心平气和、呼吸稳定。快 速节奏的音乐会使人神经系统比较兴奋, 慢速节奏的 音乐会让读者感到压抑，因此舒缓的轻音乐最适合高 校读者。调查中 $53.69 \%$ 的读者喜欢轻音乐类型的背 景音乐, 75.17\%的读者喜欢舒缓的背景音乐。开馆音 乐可选择比较振奋精神、鼓舞士气的乐曲, 让读者以 饱满的精神状态进入图书馆, 以积极向上的面貌迎接 新一天的学习和工作, 如《茉莉花》、《卡农钢琴曲》、

《拉德斯基进行曲》、理查德的《星空》、《土耳其 进行曲》、《开往春天的地铁》等; 午间音乐可选择 一些悠扬舒缓的乐曲, 给读者提供一个放松适于小䄸 的环境, 如: 《高山流水》、《乘着歌声的翅膀》、 《献给爱丽丝》、 《G大调小步舞曲》、门德尔松的 《仲夏夜之梦》和《乘着歌声的翅膀》等; 闭馆音乐, 选择一些广播提示乐曲, 使读者有一种回家的归属感, 如《Time to say goodbye》、萨卡斯音乐《回家》、 舒曼的《小夜曲等》、班得瑞的《月光水岸》等。

\section{2. 背景音乐的音量选择和时间选择}

高校图书馆背景音乐的音量选择是比较严肃的议 题。音量的大小能引起读者身心的不同感受, 引发读 者情感情绪的变化。调查问卷中有 $63.09 \%$ 的读者希 望开馆、闭馆的音量可以稍大一些，中午和课间音量 小一点。音量太大会让读者感觉身心不适, 使读者转 移注意力, 严重影响学习和阅览。音量太小, 则让读 者感受不到背景音乐的存在，亦失去其价值和意义。 适度音量的背景音乐会让读者身心愉悦, 既能享受音 乐带来的美, 又能不引起读者的大幅度情绪波动。

很多消费场所的背景音乐全天几乎不间断, 播放 时间没有限制，但高校图书馆背景音乐的播放时间应 充分考虑到读者的身心需求。调查中有 $37.58 \%$ 的读 者希望在开馆、闭馆和午间播放背景音乐，65.1\%的 读者希望每次播放时长为 10 分钟。经过调研, 很多 有背景音乐的高校图书馆播放时间大多为开馆、闭馆、 午间三个时间段, 播放频次过高会影响读者学习, 播 放频次低会失去其存在的价值和意义。

\section{4. 结语}

目前很多高校图书馆背景音乐仍在徘徊摸索阶段, 没有大胆地进行实践。适度播放背景音乐既能有助于 读者身心健康, 增强图书馆亲和力, 拉近图书馆和读 者的距离, 又能巧妙的调节读者的身心疲劳, 调研也 证明读者对适当的背景音乐持积极乐观的态度。高校 图书馆播放背景音乐不仅能为读者学习和阅览营造 
更为优雅的环境，同时无形中也能提高读者的音乐艺 术修养和审美情趣。

\section{REFERENCES}

[1] A.L. Kathleen, E.S. Dianna, Opening pandora's stream: piping music into the information literacy classroom, vol. 9, issue 2, Public Services Quarterly, 2013, pp. 89-109. https://doi.org/10.1080/15228959.2013.785876

[2] K.Liao, Analysis of the technical characteristics of Lozanov's suggestive teaching method, issue 6, Comparative Education Research, 2003, pp. 65-69. https://kns.cnki.net/kcms/detail/detail.aspx?FileNa me=BJJY200306012\&DbName=CJFQ2003 